\title{
Blunt Traumatic Pericardial Rupture with Traumatic Amaurosis Fugax Presenting as Massive Haemothorax: A Conundrum?
}

\author{
Isaac Okyere ${ }^{1 *}$, Samuel Gyasi Brenu1 ${ }^{1}$, Perditer Okyere ${ }^{2}$ \\ ${ }^{1}$ Cardiovascular and Thoracic Surgery Unit, Department of Surgery, School of Medicine \& Dentistry, College of Health Sciences, \\ Kwame Nkrumah University of Science and Technology and Komfo Anokye Teaching Hospital, Kumasi, Ghana \\ ${ }^{2}$ Department of Internal Medicine, School of Medicine \& Dentistry, College of Health Sciences, Kwame Nkrumah University of \\ Science and Technology and Komfo Anokye Teaching Hospital, Kumasi, Ghana \\ Email: ^drokyere@yahoo.com
}

How to cite this paper: Okyere, I., Brenu, S.G. and Okyere, P. (2019) Blunt Traumatic Pericardial Rupture with Traumatic Amaurosis Fugax Presenting as Massive Haemothorax: A Conundrum? Open Journal of Thoracic Surgery, 9, 9-16.

https://doi.org/10.4236/ojts.2019.92002

Received: May 14, 2019

Accepted: June 27, 2019

Published: June 30, 2019

Copyright $\odot 2019$ by author(s) and Scientific Research Publishing Inc. This work is licensed under the Creative Commons Attribution International License (CC BY 4.0).

http://creativecommons.org/licenses/by/4.0/

\begin{abstract}
Introduction: Blunt traumatic pericardial rupture (BTPR) or traumatic pericardiotomy is a rare typical trauma. It is usually discovered at autopsy. Surgical repair is mandatory especially if prompt diagnosis is made because of the associated high mortality. Clinical Case: We report the successful management and survival of BTPR patient after chest trauma presenting with massive haemothorax and transient loss of vision, necessitating urgent surgical treatment. The patient was involved in a road traffic accident having a head-on collision with the rear of the vehicle ahead whiles trying to overtake it. Discussion: It is usually discovered at autopsy or during emergent surgical exploration through either sternotomy or thoracotomy [1] due to its delayed diagnosis, unusual presentation, association with other major cardiopulmonary injuries and complications such as cardiac herniation, fatal arrhythmias, cardiogenic shock and cardiac arrest. Conclusion: Blunt traumatic pericardial rupture should be suspected in any patient in whom hemodynamic instability occurs rapidly after trauma without evidence of major bleeding. Prompt surgical exploration may yield excellent results.
\end{abstract}

\section{Keywords}

Blunt Chest Trauma, Pericardial Rupture, Haemothorax, Thoracotomy, Amaurosis Fugax

\section{Introduction}

Blunt traumatic pericardial rupture $(\mathrm{BTPR})$ or traumatic pericardiotomy is a 
rare typical trauma. It is usually discovered at autopsy [1]. Surgical repair is mandatory especially if prompt diagnosis is made because of the associated high mortality. Blunt traumatic pericardial rupture should, therefore, be suspected in all patients with severe chest injury. The key complication is cardiac herniation but it can also lead to fatal arrhythmias and cardiac arrest [2]. We report the successful management and survival of BTPR patient after chest trauma presenting with massive haemothorax and transient loss of vision as well, necessitating urgent surgical treatment.

\section{Case Report}

A 44-year-old man was rushed from a peripheral hospital with chest pains and bleeding from the face for four hours duration. Patient was well until 4 hours prior to presentation when as an unrestrained driver of a saloon car was involved in a head-on collision with the rear of the vehicle ahead whiles trying to overtake it. He did not lose consciousness, no bleeding from the ear or nose, but sustained multiple facial abrasion injuries. He had no hemoptysis, dyspnoea or abdominal pains. On admission, he was conscious and alert and well orientated to time, place and person with a temperature of $36.5^{\circ} \mathrm{C}, \mathrm{BP}$ of $100 / 66 \mathrm{mmHg}$, heart rate of $106 \mathrm{bpm}$ of moderate volume and a respiratory rate of 29 cycles $/ \mathrm{min}$.

Chest expansion was reduced on the left hemithorax, with dull percussion note and reduced air entry. The abdomen was full, soft and non-tender with normal bowel sounds. He could move all extremities with no tenderness or deformity.

Extended Focused Assessment with Sonography for Trauma (eFAST) was positive for left pleural space collection but negative for haemoperitoneum and haemopericardium. Diagnosis of chest trauma with left Pneumohaemothorax and multiple facial abrasion secondly to road traffic accident was made. So the patient was started with Intravenous fluid with a litre of Ringers lactate run fast, antibiotics, i.v cefuroxime $1.5 \mathrm{~g}$ start, and blood was taken for complete blood count, chemistry, kidney and liver function tests. Serum was also group-match against 4 units of blood. Antitetanus prophylaxis, i.m tetanus toxoid, ATS 1500 UI doses given and a size 36 FG chest tube was passed under local infiltration draining immediately $1200 \mathrm{mls}$ of blood and the tube was clamped. Another 700 mls of blood was drained after an hour when the clamp was released, therefore a massive haemothorax diagnosis was made and the patient was then prepared for surgery. Chest $x$-ray after the chest tube insertion showed a left lower opacification with pleural effusion as shown in Figure 1. As patient being prepared for surgical exploration, he suddenly became totally blind, so an urgent CT head scan was requested and the neurosurgeons and ophthalmology surgical teams consulted. However, he had remained stable after two units of whole blood transfusion. The CT scan of the Head showed basal skull fracture with bilateral occipito-parietal, non-hemorrhagic contusions as shown in Figure 2 and the neurosurgeons recommended a conservative management upon review. However after 


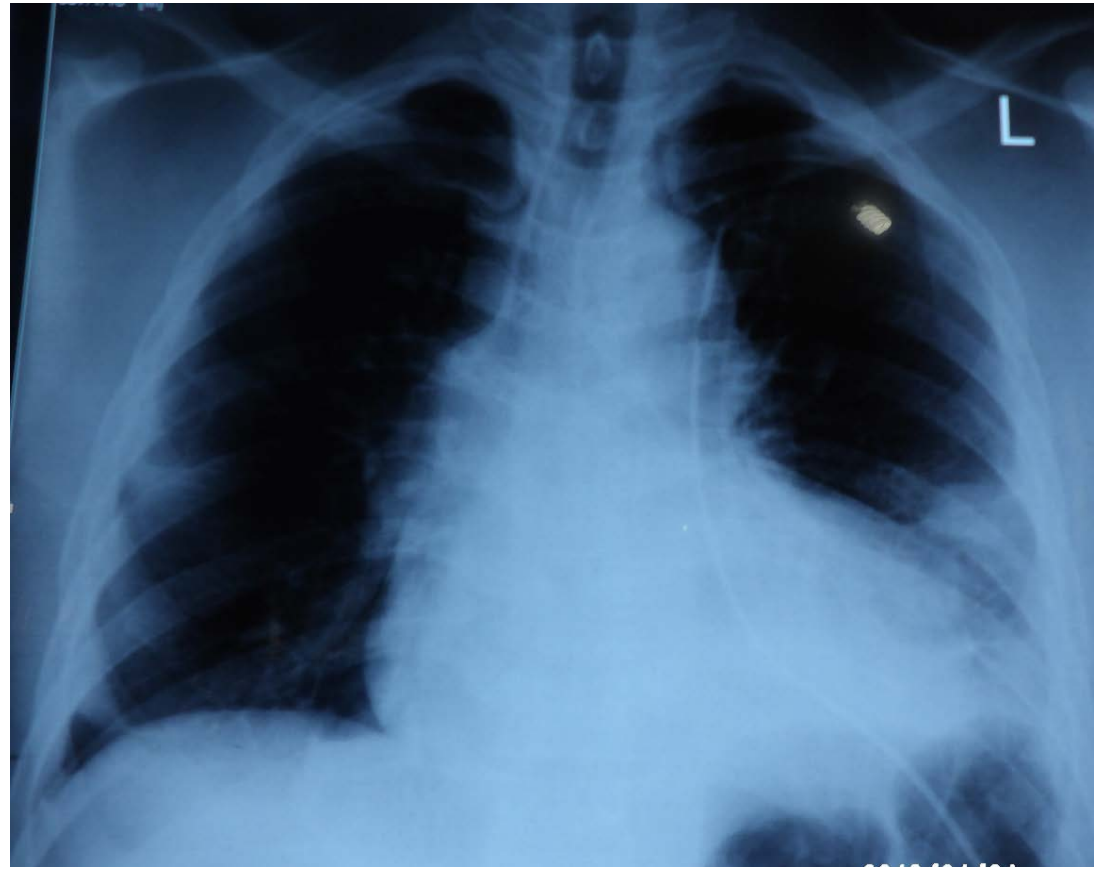

Figure 1. Chest $\mathrm{x}$-ray showing left lower opacification with a chest tube in-situ.

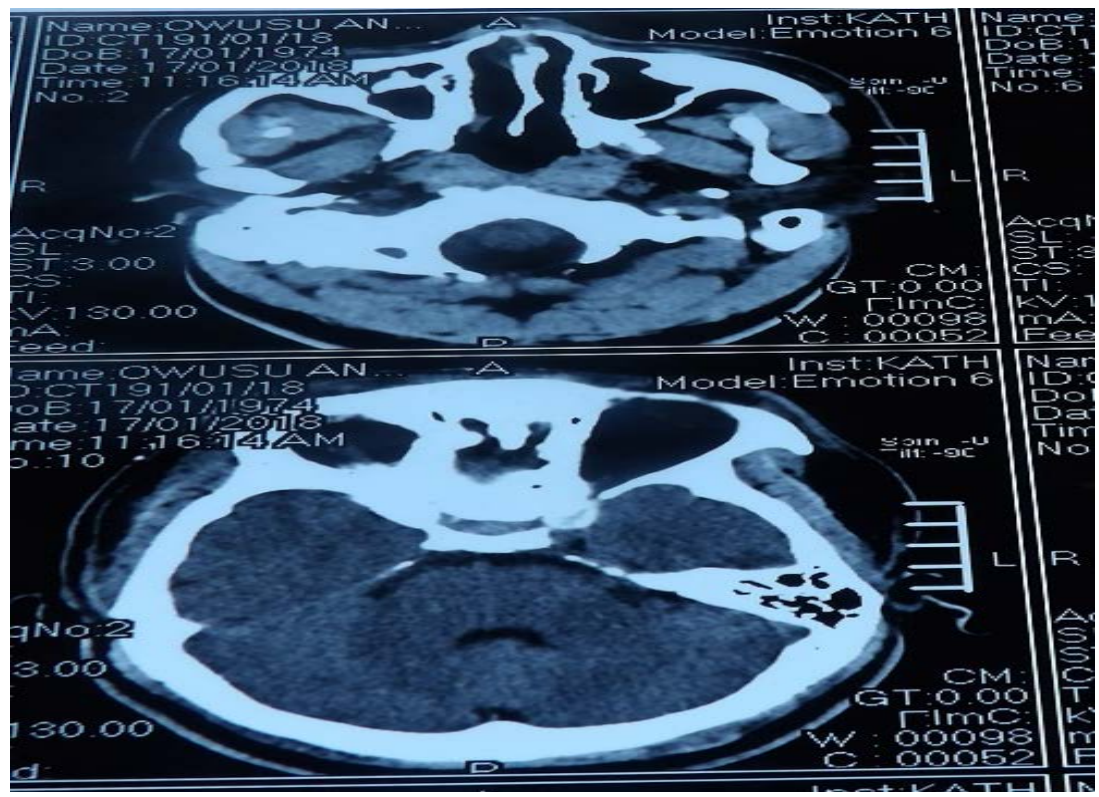

Figure 2. Head CT Scan showing basal skull fracture with bilateral occipito-parietal, non-hemorrhagic contusion.

eight hours before the ophthalmologist could review on the morning of surgery, he had fully regained his sight and the ophthalmologist review showed that the vision was normal for counting figures in both eyes, he had normal bilateral anterior segment and normal fundus examination and therefore made a provisional diagnosis of transient visual impairment possibly from cerebral contusion and also recommended like the neurosurgeon a conservative management and for a follow up after surgery. He was then taken to the operating theatre. 
His laboratory investigations before surgery showed an $\mathrm{Hb}$ of $9.0 \mathrm{~g} / \mathrm{dl}$ after the 2 units of whole blood transfusion, White cell count of 15.80, Platelets of 159 and Neutrophils of $81 \%$. Urea of $5.46 \mathrm{mmol} / \mathrm{l}(2.5-8.30)$ and creatinine: 131 umol/l (44 - 80), Na: $138 \mathrm{mmol} / \mathrm{l}, \mathrm{K}: 4.8 \mathrm{mmol} / \mathrm{l}, \mathrm{CL}: 112 \mathrm{mmol} / \mathrm{l}$, AST: 565.1 U/L, ALT: $575.1 \mathrm{U} / \mathrm{L}$, ALP: $36.8 \mathrm{U} / \mathrm{L}, \mathrm{GGT}: 101.6 \mathrm{U} / \mathrm{L}$, total protein: $43.6 \mathrm{~g} / \mathrm{l}$ and albumin: $27.7 \mathrm{~g} / \mathrm{l}$.

In the operating theatre, under general anaesthesia and double lumen intubation with size 39FG for isolation of the left lung and in right lateral decubitus position, a left standard posterolateral thoracotomy was performed entering the 7 th intercostal space or pleural bed. There was approximately $900 \mathrm{mls}$ of collected pleural blood, complete laceration of the pericardium extending from the diaphragmatic surface to the aortic root as shown in Figure 3, complete and thrombosed transection of the left pericardiophrenic artery, but intact coronary arteries and the coronary veins. The artery was ligated, the margins of the pericardium coagulated. Haemostasis was secured, the thoracic cavity irrigated with 1 litre of warm normal saline, a size 32 FG chest tube placed and the chest closed up in layers. The patient was extubated on table. He had uneventful course postoperatively with the removal of chest tube on the $3 \mathrm{rd}$ postoperative day and was discharged on the 8th postoperative day. He was followed-up at 2 weeks, one month and after 3 months concomitantly by the neurosurgeon, the ophthalmologist and at our clinic and has been well without any sequelae with intact vision, carrying out his daily activity.

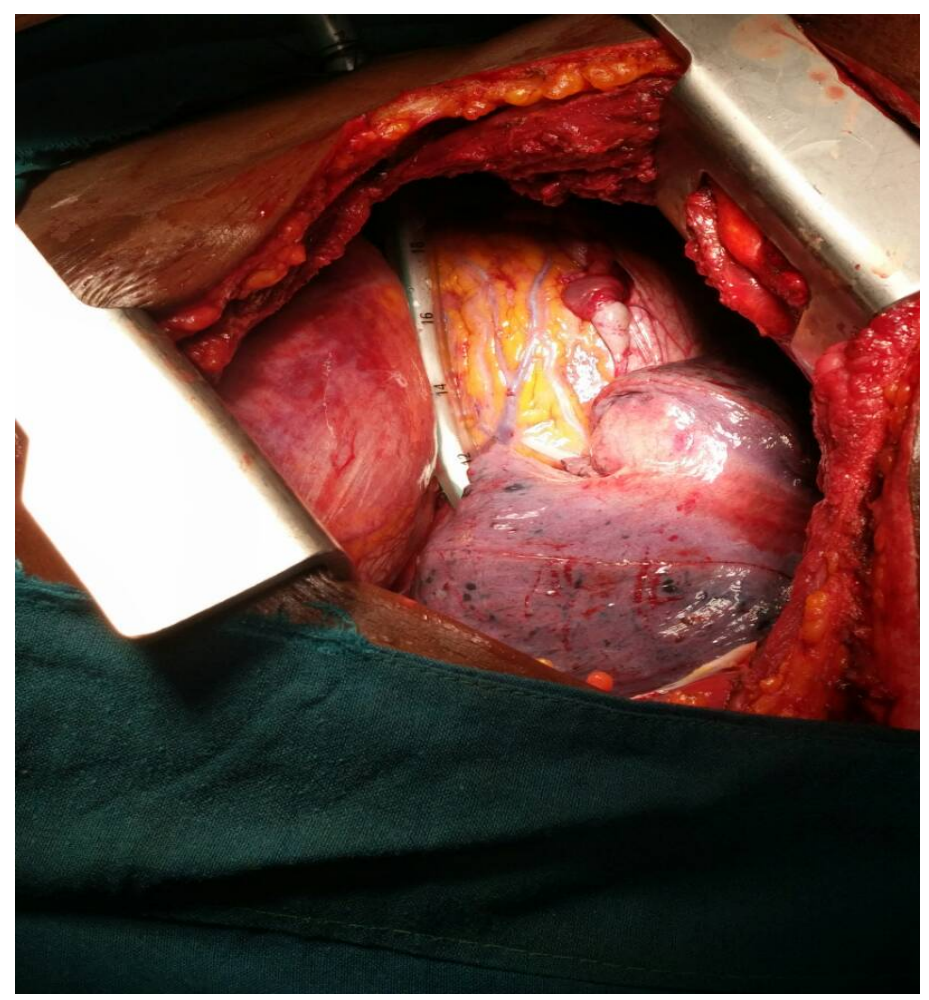

Figure 3. Intraoperative picture showing the heart, the diaphragm, the edge of the ruptured pericardium with exposed vessels, chest tube and collapsed left lung. 


\section{Discussion}

Blunt traumatic pericardiotomy is a very rare condition documented to be seen in $0.1 \%$ of cases of chest trauma [3] [4] and in $0.5 \%$ of patients who present with blunt chest trauma [5]. It has been found to occur most commonly in the left pleuropericardial border in multiple literatures with other documented areas of occurrence being the diaphragmatic, right pleuropericardial and superior mediastinal borders in descending order [3] [4] [5] [6]. Right pleuropericardial tears can cause herniation of the heart into the right hemithorax and potentially result in torsion of the great vessels, a situation which is potentially fatal due to its lethal hemodynamic effect. Consistent with these observations, our patient's pericardial tear occurred on the left. Diaphragmatic tears can result in herniation into the abdomen or vice versa [5]. It is most commonly caused by fallout from high-velocity collisions as was our case, but few cases have been reported to have occurred from a fall from height [3] [5]. Due to the associated high energy impact, there are usually other associated injuries. Kittaka and colleagues reported that up to about $40 \%$ of patients have associated myocardial injuries [4] while extremity fractures, abdominal and neurologic injuries have been reported as associated injuries [5]. It is associated with a high mortality rate of over $50 \%$ [7].

\section{Clinical Presentation}

Clinical presentation usually depends on the associated organ injuries due to the trauma [3]. However, in the event of an isolated traumatic pericardiotomy, the clinical picture of the patient is dependent on the aperture of the tear. Tears between 8 and $12 \mathrm{~cm}$ can cause a pericardial aperture just big enough to cause herniation and strangulation of the heart thereby presenting as a case of cardiac tamponade. Larger tears may not strangulate the heart as was this patient and as such may be asymptomatic only to be picked up as an incidental finding in exploratory thoracotomy for patients with multiple injuries [8] [9]. Our patient sustained a large left pericardiopleural tear with massive haemothorax necessitating chest tube insertion and the subsequent emergency exploratory thoracotomy. Cardiac herniation is the most common complication of traumatic pericardiotomy and has been reported to have a mortality rate of about $75 \%$ mainly due to the concomitant hemodynamic instability [10]. However, despite the high mortality associated with traumatic pericardial rupture, patients may survive it if prompt diagnosis and surgical intervention is taken. Other documented complications include torsion of the great vessels, arrhythmias and massive hemothorax [3] [4] [5] [9]. Massive hemothorax either due to an isolated pericardial tear or a combination of myocardial and pericardial tears as was documented in the case report by Kittaka et al., is also a significant cause of mortality due to the potential for hemorrhagic shock or cardiopulmonary arrest [4]. The patient suffered massive haemothorax from the pericardial tear and pericardiophrenic artery injury. 


\section{Diagnosis}

Options for radiological imaging of the heart and pericardium in acute trauma include ultrasonography in the form of FAST (Focused Assessment with Sonography in Trauma) or echocardiography, chest radiography and CT scan. FAST has been reported to have a high sensitivity $(97 \%-100 \%)$ in identifying pericardial injuries [11]. Chest $\mathrm{x}$-rays have limited potentials to identify pericardial or cardiac injuries. However, it is particularly useful in identifying associated complications such as hemothorax, pneumothorax, pneumomediastinum or pneumopericardium [11]. Most commonly identified radiological features seen on chest $\mathrm{x}$-ray are deviation of pericardial shadow and pneumopericardium [5]. CT scan of the chest is the preferred radiological technique in diagnosing traumatic pericardiotomy which characteristically shows as empty pericardial sac sign, pneumopericardium or pericardial displacement [7] [10]. Despite these available radiologic interventions, the non-specificity of clinical signs and symptoms suggestive of traumatic pericardiotomy and the severe hemodynamic instability result in prioritization of surgical exploration as against further radiological evaluation hence an in intra-operative diagnosis [7] [12]. The initial diagnostic imaging done for our patient was the EFAST, because he was unstable and this was able to pick the haemothorax. A chest $\mathrm{x}$-ray was done after patient's stabilization including the passage of a tube thoracostomy.

\section{Treatment}

The options of management of traumatic pericardiotomy as reported in several literatures include non-closure of defect, primary closure with nonabsorbable sutures, closure via mesh repair and complete pericardiotomy [3] [5] [10] [12]. Verkroost and colleagues reported that pericardial ruptures with little potential of cardiac herniation do not require surgical closure [3]. Tears ranging from 8 to $12 \mathrm{~cm}$ have been found to have a potential for cardiac herniation and as such should be repaired either by primary closure or mesh repair. However, complete pericardiotomy in such situations is highly recommended by some literatures so as to prevent cardiac tamponade and associated cardiogenic shock which may arise from cardiac swelling following primary closure and this is what was done for our patient because of the large tear. Complete pericardiotomy is also particularly useful in patients with concomitant myocardial contusions and complications of arrhythmias [7] [12]. A retrospective review by Wang and Li reported that over $70 \%$ of cases of traumatic pericardial rupture were repaired by the use of synthetic grafts such as Goretex, Dacron or mesh, while complete pericardiotomy was done in about 5\% of them. In $9.5 \%$ of these 42 cases reviewed, no repair was done [12]. In the present case, we left the large pericardial rupture intact after haemostasis. Our patient had uneventful surgery and postoperative course because of effective resuscitation and early surgical intervention. Therefore traumatic pericardial rupture should be suspected in all severe chest trauma patients. 


\section{Conclusion}

Blunt traumatic pericardial rupture should be suspected in any patient in whom hemodynamic instability occurs rapidly after trauma without evidence of major bleeding. On most occasions, there is no time for additional diagnostic measures other than EFAST, chest x-ray and chest CT scan if available, and the patient condition tolerates it. Prompt surgical exploration may yield excellent results.

\section{Acknowledgements}

We are grateful to the theatre staff of the accident and the emergency centre of the Komfo Anokye Teaching Hospital and to the patient for consenting for the publication of the case.

\section{Conflicts of Interest}

The authors declare no conflicts of interest regarding the publication of this paper.

\section{References}

[1] Wall, M.J., Mattox, K.L. and Wolf, D.A. (2005) The Cardiac Pendulum: Blunt Rupture of the Pericardium with Strangulation of the Heart. Journal of Trauma, Injury, Infection, and Critical Care, 59, 136-141. https://doi.org/10.1097/01.TA.0000171466.47885.C5

[2] Fulda, G., Brathwaite, C.E., Rodriquez, A., Turney, S.Z. and Cowley, R.A. (1991) Blunt Traumatic Rupture of the Heart and Pericardium: A Ten-Year Experience (1979-1989). Journal of Trauma, Injury, Infection, and Critical Care, 31, 167-173. https://doi.org/10.1097/00005373-199131020-00003

[3] Verkroost, M.W. and Hensens, A.G. (1998) Isolated Pericardial Rupture with Left-Sided Haematothorax after Blunt Chest Trauma. European Journal of Cardio-Thoracic Surgery, 14, 517-519. https://doi.org/10.1016/S1010-7940(98)00235-8

[4] Kittaka, H., Yagi, Y., Zushi, R., Hazui, H. and Akimoto, H. (2015) Combination of Blunt Cardiac and Pericardial Injury Presenting a Massive Hemothorax without Hemopericardium. Acute Medicine \& Surgery, 2, 257-259.

https://doi.org/10.1002/ams2.112

[5] Chughtai, T., Chiavaras, M.M., Sharkey, P., Shulman, H. and Miller, H.A. (2008) Pericardial Rupture with Cardiac Herniation. Canadian Journal of Surgery, 51, E101-E102.

[6] Kamiyoshihara, M., Igai, H., Kawatani, N. and Ibe, T. (2016) Right or Left Traumatic Pericardial Rupture: Report of a Thought-Provoking Case. Annals of Thoracic and Cardiovascular Surgery, 22, 49-51. https://doi.org/10.5761/atcs.cr.15-00142

[7] Dato, G.M.A., Arslanian, A., Filosso, P.L., Aidala, E., Adduci, M., Bardi, G., et al. (2002) Heart Herniation after Blunt Chest Trauma. The Journal of Thoracic and Cardiovascular Surgery, 123, 367-368. https://doi.org/10.1067/mtc.2002.119879

[8] Rippey, J.C.R., Rao, S. and Fatovich, D. (2004) Blunt Traumatic Rupture of the Pericardium with Cardiac Herniation. Canadian Journal of Emergency Medicine, 6, 126-129. https://doi.org/10.1017/S1481803500009106

[9] Sohn, J.H., Song, J.W., Seo, J.B., Do, K.H., Lee, J.S., Kim, D.K., et al. (2005) Pericardial Rupture and Cardiac Herniation after Blunt Trauma: A Case Diagnosed Using Cardiac MRI. The British Journal of Radiology, 78, 447-449. 
https://doi.org/10.1259/bjr/31146905

[10] Hoyos, J.P., Wolff, J.D., Cuevas, L. and Carlos, H. (2017) A Case of Cardiac Herniation after Thoracic Blunt Trauma. Journal of Trauma and Care, 3, 2-5.

[11] Adams, A., Fotiadis, N., Chin, J.Y., Sapsford, W. and Brohi, K. (2012) A Pictorial Review of Traumatic Pericardial Injuries. Insights Imaging, 3, 307-311. https://doi.org/10.1007/s13244-012-0177-9

[12] Wang, H. and Li, M. (1995) Blunt Traumatic Pericardial Rupture. Journal of Accident \& Emergency Medicine, 12, 55-56. https://doi.org/10.1136/emj.12.1.55 